V. Ewerbeck

S. Sell

\title{
Vor dem Deutschen Orthopädenkongress 2004
}

\author{
Before the Annual Meeting of the German Orthopedic Societies 2004
}

\section{Verehrte Leserinnen und Leser,}

es ist gute Tradition, dem Präsidenten der DGOOC in der Zeitschrift für Orthopädie im Vorfeld des jährlichen Deutschen Orthopädenkongresses Raum für ein Editorial zur Verfügung zu stellen. Die gleiche Tradition gibt es bei den „Orthopädie-Mitteilungen“ für den Präsidenten der DGOOC gemeinsam mit dem Tagungspräsidenten des BVO. Dass sich die wissenschaftliche Fachgesellschaft und der Berufsverband aus guten Gründen strukturiert aufeinander zubewegen und kurz davor stehen, einen gemeinsamen Kernvorstand $z u$ institutionalisieren, dürfte vielen von Ihnen bekannt sein. Entsprechende Satzungsänderungen, die von den Mitgliedschaften abgestimmt werden müssen, sind in Vorbereitung. Dies geschieht in der Erkenntnis, dass die Interessen von uns Orthopäden trotz unterschiedlichen beruflichen Alltages in Praxis und Klinik nur gemeinsame sein können. Sicher gibt es graduelle Unterschiede. Ein Prinzip aber gilt: Was den einen stark macht, stärkt auch den anderen, was dem Einen schadet, schwächt auch den Anderen. Hier gibt es keinen Zweifel. Und weil das so ist, ergibt sich folgerichtig, dass die Botschaften vor dem Kongress sowohl in der Zeitschrift für Orthopädie als auch in den Orthopädiemitteilungen identisch sein müssen. Die Zielgruppen mögen differieren, die Interessen decken sich. Und damit auch unsere Aufgaben. Sehen Sie es also bitte nicht als Folge der Bequemlichkeit oder der Fantasielosigkeit des Präsidenten, dass nicht zwei verschiedene Editorials in Druck gehen. Es ergibt sich einfach aus unserer Realität.

\section{Mit herzlichen Grüßen}

Professor Dr. med. Volker Ewerbeck Präsident der DGOOC

Professor Dr. med. Stefan Sell Tagungspräsident BVO
Die bevorstehende Zusammenführung der Weiterbildungsordnungen von Orthopäden und Unfallchirurgen und damit die enge Verknüpfung zweier bisher getrennter Fächer hat bei allen Beteiligten Denkprozesse zur Definition des eigenen Berufes, zum eigenen Selbstverständnis und zum Standort des jetzigen und des künftigen Berufsbildes im gesellschaftlichen Umfeld ausgelöst. Dies war und ist ein außerordentlich kreativer Vorgang. Vielen von uns wurden Dinge bewusst, die wir als selbstverständlich hingenommen haben, dies aber nicht hätten tun sollen. Defizite und Stärken traten gleichermaßen klar hervor. Und klar wurde auch, was bei allem Veränderungsdruck bleiben muss, was zählt. Es ist eben nicht unsinnig, uns selbst, unseren Patienten, politischen und wirtschaftlichen Entscheidungsträgern und nebenbei auch unseren unfallchirurgischen Freunden klar zu machen, von was sie reden, wenn sie den Begriff „Orthopädie“ verwenden:

„Orthos“ heißt gerade und „pais“ heißt das Kind. Vom Ursprung her kümmert sich Orthopädie darum, dass Kinder sich richtig entwickeln, sich richtig bewegen, richtig wachsen. Orthopädie ist die medizinische Kunst, die sich um die zuverlässige Funktion dessen kümmert, was ein zentraler Teil des menschlichen Selbstverständnisses ist, ohne dass wir uns dessen immer bewusst sind: nämlich, dass wir uns bewegen können. Was macht den Menschen zum Menschen? Jeder empfindet reflexartig Folgendes: die Tatsache, dass er denken kann, dass er ein Bewusstsein seiner selbst hat, dass er sprechen kann. Die Fähigkeit, sich bewegen zu können, erscheint auf den ersten Blick in Bezug auf das Selbstverständnis geringerwertig. Auch Affen können sich bewegen, sogar besser als Menschen. Nur: Nehmen Sie dem Menschen seine Bewegungsmöglichkeit, lassen Sie ihn Schmerzen bei jeder Bewegung haben, lassen Sie ihn durch unkoordinierte Muskelaktionen bei jedem Schritt mit sich selbst kämpfen, lassen Sie das Kind wegen zunehmender Wirbelsäulendeformierung langsam Atemnot bekommen, lassen Sie den alten Menschen wegen zunehmender 
Gelenkeinsteifung schließlich vereinsamen, lassen Sie alle diese Menschen, die bloß diese niederwertige Fähigkeit des Sich-Bewegen-Könnens verloren haben, oder nie richtig hatten, einmal darüber sprechen, wie sie sich fühlen. Fast alle fühlen sich einer Grundqualität des Menschseins beraubt.

Es gibt zahllose Ursachen, sich nicht mehr richtig bewegen zu können. Eine Verletzung ist nur eine davon. Orthopädie beschäftigt sich mit allen.

Der öffentlichen Hand in der Bundesrepublik fehlt Geld an allen Ecken. Also muss an allen Ecken entweder Geld besorgt oder Geld eingespart werden. Überall gilt es zwischen diesen beiden Varianten zu entscheiden oder einen Mittelweg zu finden. Die Frage lautet stets: Was wird wirklich gebraucht? Auch wir müssen uns diese Frage stellen lassen. Naturgemäß beantworten wir sie zunächst folgendermaßen: „Wir werden gebraucht“. Auch wenn Sie damit Recht haben, liebe Kolleginnen und Kollegen, wir müssen es belegen. Die Forderung nach einem Beweis der eigenen Existenznotwendigkeit erscheint angesichts von überfüllten Praxiswartezimmern, Wartelisten vor elektiven orthopädisch-chirurgischen Eingriffen und anderen Indikatoren geradezu lachhaft. Sie ist es aber nicht. Aus der Tatsache, dass Erkrankungen und Verletzungen des muskuloskelettalen Systems 42\% aller Arbeitsunfähigkeitstage in Deutschland verursachen, dass $30 \%$ der über fünfzigjährigen Frauen und mehr als 20\% der über fünfzigjährigen Männer mindestens einmal jährlich einen Facharzt für Orthopädie aufsuchen, dass $42 \%$ aller Rehabilitationsleistungen und 30\% aller Berentungen auf Folgen von Erkrankungen der Haltungs- und Bewegungsorgane zurückzuführen sind, ergibt sich noch lange nicht, dass dies so sein muss, dass dies alles notwendig ist. So jedenfalls sieht es die Politik, und dieser Sichtweise müssen wir uns stellen. Alles was wir tun, muss lückenlos als notwendig bewiesen werden und die Ergebnisse dessen was wir tun, müssen nachprüfbar gut, besser noch exzellent sein. Und schließlich brauchen wir in unserer Mediengesellschaft Leute unter uns, die diese Botschaften aus innerster Überzeugung heraus auf möglichst vielen Ebenen kommunizieren. Wir brauchen für diese Aufgabe nicht wenige, wir brauchen viele Leute. Wir leben nicht nur in einer Mediengesellschaft, wir leben auch in einer Kommissionsgesellschaft. Es ist nachgerade unfassbar, wie viele Gremien, wie viele Kommissionen, wie viele Ausschüsse es gibt, die über Belange der muskuloskelettalen Medizin entscheiden, ohne dass unsere ärztliche Kompetenz dort angemessenen Einfluss hätte. Es stimmt - Gremienarbeit ist lästig, zeitraubend, nervtötend ... aber gemacht werden muss sie. Und zwar von möglichst vernünftigen Menschen. Von Menschen, die wissen, wann Evaluation beginnen muss und auch wann sie aufhören muss. Wann die Forderung nach einem Nachweis evidenzbasierter Entscheidungsgrundlagen sich in das Gegenteil dessen verkehrt, was sie anzustreben vorgibt. Es ist z.B. schlicht unsinnig, evidenzbasiert nachweisen $\mathrm{zu}$ wollen, dass auch auf orthopädisch-chirurgischem Gebiet Übung den Meister macht. Jeder weiß, dass es so ist, und jeder weiß, dass ein Nachweis nach Evidenzkriterien allein aus ethnischen Gründen nicht möglich ist. Diesen Nachweis, wie geschehen, beispielsweise für den Bereich der Kniegelenksendoprothetik zu fordern, beruht nicht auf dem Wunsch nach kristallklarer wissenschaftlicher Wahrheit und auch nicht auf dem Wunsch nach dem Wohle des Patienten oder nur dessen Recht zur Entscheidungsfreiheit. Er beruht ganz einfach auf wirtschaftlichen Eigeninteressen. Auch solche Fragen werden in Kommissionen entschieden, in denen wir entweder Sitz und Stimme haben oder eben nicht.

Wir werden mit unserem Kongress den politischen, den ökonomischen, den gesellschaftlichen Mainstream nicht maßgeblich beeinflussen können. Wir können und werden uns aber deutlich und vernehmlich äußern. Dass auf die muskuloskelettale Medizin ein Nachfrageschub ohnegleichen zukommt, möge sich jeder klar machen. Dass die Alterspyramide zwischenzeitlich Kopf steht, ist hinlänglich bekannt. Ebenso, dass heute jedes zweite neugeborene Mädchen über eine Lebenserwartung von $100 \mathrm{Jah}-$ ren verfügt. Oder wussten Sie das nicht? Dies bedeutet unter anderem, dass in absehbarer Zeit sehr viele Menschen unter uns leben werden, deren Gelenke länger in Betrieb waren, als sie das schadlos überstehen können. Dies ist nur ein Beispiel. Es gilt jedoch, uns und anderen vor Augen zu halten, wie unsere Gesellschaft mit diesen Zahlen und diesen von niemandem angezweifelten Prognosen umgeht: Die international vorliegenden Daten der Bone and Joint Decade haben in den Vereinigten Staaten von Amerika dazu geführt, dass eine erhebliche Ausweitung des Lehrangebotes für Studenten und weiterzubildende Ärzte auf dem Gebiet der Orthopädie und der Orthopädischen Chirurgie erfolgen soll. In Deutschland wird der umgekehrte Weg beschritten. Wie viele der ehemals 37 Lehrstühle für das Fach Orthopädie in Deutschland in 5 Jahren noch übrig sind, ist völlig offen. Vielleicht werden es nur 20 sein. Wie Ausbildung, Weiterbildung, Lehre und Forschung unter diesen Bedingungen aufrecht erhalten werden sollen, und wie Versorgungsengpässe angesichts der auf uns zukommenden Nachfragensituationen vermieden werden sollen, ist rätselhaft. Die geplante, vom Bundesärztetag empfohlene und von zahlreichen Landesärztetagen bereits abgesegnete Zusammenführung der Weiterbildungsordnung der Fächer Orthopädie und Unfallchirurgie wird vielerorts zum Anlass genommen, Stellenstreichungen als Zusammenführung zu verkleiden. Für Krankenhausträger und Fakultäten bedeutet diese verführerische Einsparmöglichkeit eine Vorentscheidung: Aus Zwei machen wir Einen, und wer das sein soll, entscheidet der Kostenträger. Nicht die Expertise, nicht die Breite der Kenntnisse, nicht der aktuelle Bedarf und nicht die Bedarfsprognose, es entscheidet die Chance, Geld einzusparen. Und es entscheidet die Lautstärke der Selbstdarstellung. Die universitäre Medizin ist davon leider nicht ausgenommen, sie ist in fataler Weise geradezu Vorreiter dieser Entwicklung. Die Umsetzung des Prinzips „aus Zwei mach Einen“ wird das neue Fach schwächen und nicht wie gewünscht durch Bündelung von was auch immer stärken. Das betrifft Orthopäden und Unfallchirurgen. Der niedergelassene Bereich ist von dieser Entwicklung mit nur geringer Verzögerung ebenso hart betroffen: Von wo soll der Nachwuchs kommen?

Lassen Sie uns, liebe Kolleginnen und Kollegen, den bevorstehenden Kongress nutzen. Lassen Sie uns nach innen und nach außen zeigen, was zählt: Das Motto eines großen Wirtschaftbetriebes gilt auch für uns: Messen, Zählen, Wiegen, Überleben. Wir werden zeigen, dass beste Qualität von uns kommt. Und wir werden zeigen, auf welchem Fundament das möglich ist. Das Kongressmotto fasst alles zusammen. Und wir werden auf unserem Schiff auf rauher See zeigen: In der Orthopädie sind „all hands on deck“.

Wir freuen uns darauf, Sie in Berlin begrüßen zu können. 